\title{
THE MANY FUTURES OF ACTIVE DEBRIS REMOVAL
}

\author{
Adam E. White ${ }^{a^{*}, H_{u g h} \text { G. Lewis }}{ }^{\mathrm{a}}$ \\ ${ }^{a}$ Astronautics Research Group, Faculty of Engineering and the Environment, University of Southampton, \\ Southampton, SO17 1BJ, United Kingdom, \\ Adam E. White email: adam.white@ southampton.ac.uk \\ Hugh G. Lewis email: hglewis@soton.ac.uk
}

*Corresponding author email: adam.white@ southampton.ac.uk

Tel: +442380 597658, Fax: +442380593058

\begin{abstract}
In the last decade, space debris modelling studies have suggested that the long-term low Earth orbit (LEO) debris population will continue to grow even with the widespread adoption of mitigation measures recommended by the Inter-Agency Space Debris Coordination Committee. More recently, studies have shown that it is possible to prevent the expected growth of debris in LEO with the additional removal of a small number of selected debris objects, through a process of active debris removal (ADR). In order to constrain the many degrees of freedom within these studies, some reasonable assumptions were made concerning parameters describing future launch, explosion, solar and mitigation activities. There remains uncertainty about how the values of these parameters will change in the future. As a result, the effectiveness of ADR has only been established and quantified for a narrow range of possible future cases. There is, therefore, a need to broaden the values of these parameters to investigate further the potential benefits of
\end{abstract} ADR.

A study was completed to model and quantify the influence of four key parameters describing launch and explosion rates, the magnitude of solar activity and the level of post-mission disposal compliance on the effectiveness of ADR to reduce the LEO debris population. Each parameter's value was drawn from a realistic range, based upon historical data of the last 50 years and, in the case of post-mission disposal, a current estimate of the level of compliance and a second optimistic value. Using the University of Southampton's Debris Analysis and Monitoring Architecture to the Geosynchronous Environment (DAMAGE) model, the influence of each parameter was modelled in Monte Carlo projections of the $\geq 5 \mathrm{~cm}$ LEO debris environment from 2009 to 2209. In addition, two ADR rates were investigated: five and ten removals per year. 
The results showed an increase in the variance of the size of the LEO population at the 2209 epoch compared with previous ADR modelling studies. In some cases, the number of LEO debris objects in the population varied by a factor greater than ten. Ten removals per year were not sufficient to prevent the longterm growth of the population in some cases, whilst ADR was not required to prevent population growth in others.

Keywords: Space debris; Orbital Debris; Remediation; Orbital Debris Removal; Active Debris Removal; ADR;

\section{Introduction}

It is well known that space debris represents a significant collision risk to operational satellites as well as a threat to the long-term sustainability of outer space activities. Several responses outlining mitigation procedures, including the Inter-Agency Space Debris Coordination Committee (IADC) Space Debris Mitigation Guidelines [1], the United Nations Committee on the Peaceful Uses of Outer Space Mitigation Guidelines [2], the International Organization for Standardization Space Debris Mitigation standards [3], the ESA Space Debris Mitigation Handbook [4], and a multitude of other national and international documents have been, and continue to be, developed to limit the expected future growth of the debris population. Whilst the widespread adoption of mitigation measures has been shown to be effective at reducing this predicted growth [5-7], these are unlikely to stop the long-term debris population in low Earth orbit (LEO) from increasing in size [8-10]. In 2009, the IADC initiated an action item (A.I. 27.1) [11] to determine the stability of the future LEO environment. Using six agencies' modelling capabilities, a consensus was reached that confirmed, even with high levels of mitigation (90\% future compliance with a 25 -year post-mission disposal (PMD) rule and no future explosions), the current LEO debris population is likely to grow. As such, one of the key conclusions of this action item was that more aggressive measures, such as active debris removal (ADR), should be considered.

Active debris removal studies performed by the National Aeronautics and Space Administration (NASA) Orbital Debris Program Office [12], the International Academy of Astronautics [13], the University of Southampton [14] and many others, have demonstrated the effectiveness of ADR in reducing the predicted LEO population. Results have shown that it may be possible to reduce the growth of the $\geq 10 \mathrm{~cm}$ LEO population by removing a number of target debris objects alongside widespread compliance with IADC mitigation guidelines. These studies have demonstrated that removal rates in the order of five objects per year may be sufficient to address the growth of the LEO population $\geq 10 \mathrm{~cm}$ over a 200 -year period [12].

To constrain the many degrees of freedom in these ADR studies some reasonable assumptions were made that confined parameters including the future launch, explosion and mitigation activity to a limited 
number of cases. The values of these parameters remained unchanged throughout the studies, for example eight-year launch traffic cycles, repeating solar cycle projections, a fixed level of PMD compliance and no explosions. In recognition of this, some authors of these studies have stated that calculated removal rates are only intended to serve as a guide, in particular

“The 'removing five objects per year can stabilize the LEO environment conclusion' is somewhat notional. It is intended to serve as a benchmark for ADR planning." (J.C. Liou, June 2012, Presentation at the 2nd European Workshop on Active Debris Removal, CNES HQ, France, slide 19) [15]

Consequently, because of the restricted range of these parameters, the effectiveness of ADR has only been investigated for a narrow range of possible future cases. Several previous modelling studies [16-19] have shown that adjusting the values of these parameters, such as increasing or decreasing launch rates or modifying solar cycle projections, can significantly influence the size of the future debris population. Whilst these previous studies have investigated variations in individual parameters, they have not considered the effects of their variations in conjunction with ADR activities. There remains a need to model a broader range of values for these parameters to help establish ADR removal rates in a wider context.

In this work, four key modelling parameters were varied; these were launch and explosion rate, magnitude of solar activity and compliance with PMD. To investigate these parameters, with respect to the effectiveness of ADR, the University of Southampton's evolutionary model, DAMAGE (the Debris Analysis and Monitoring Architecture for the Geosynchronous Environment) was used to simulate the future $\geq 5 \mathrm{~cm}$ LEO debris population over 200 years.

At the beginning of each Monte Carlo (MC) projection, four uniformly distributed random numbers were generated. These numbers dictated the future launch rate, magnitude of solar activity, level of compliance with PMD and explosion rate for that particular projection. The range of each parameter's value, excluding PMD compliance, was derived from the maximum and minimum values of the last 50 complete years of historical data. The range of PMD compliance was between an estimate of the current level of PMD and an optimistic level that may be achievable in the future. Throughout the projection, the value of each parameter remained fixed. Thus, each projection contained a different set of future conditions throughout its projection period.

To demonstrate the effect these parameters had on ADR activities, two ADR scenarios and a baseline scenario with no ADR were investigated. To capture a wide variety of possible cases, $200 \mathrm{MC}$ projections were conducted for each scenario.

\section{Method}


A 200-year future projection from 1 May 2009 to 1 May 2209 for the effective LEO debris population was used by DAMAGE. The description of this study is shown in Table 1. Three scenarios were investigated using Monte Carlo (MC) simulation technique that comprised of 200 future projections. These were the removal of five objects per year (ADR5), the removal of 10 objects per year (ADR10) and a scenario with no ADR (ADR0).

Table 1. Summary description of the study.

\begin{tabular}{|c|c|}
\hline Parameter & Value \\
\hline tion & $\begin{array}{l}\text { Meteoroid and Space Debris Terrestrial Environment Reference (MASTER) } \\
\text { population } \geq 5 \mathrm{~cm} \text { residing in or passing through the LEO regime on } 1 \text { May } 2009\end{array}$ \\
\hline Sources included & Satellites, rocket bodies, mission-related debris, explosion and collision fragments \\
\hline Sources excluded & $\begin{array}{l}\text { Reusable launch systems, space stations, new solid rocket motor slag }\left(\mathrm{Al}_{2} \mathrm{O}_{3}\right) \text {, } \\
\text { sodium potassium droplets (NaK), ejecta and paint flakes }\end{array}$ \\
\hline $\begin{array}{l}\text { Satellite } \\
\text { properties }\end{array}$ & $\begin{array}{l}\text { The operational lifetime of satellites was set to eight years, no station keeping or } \\
\text { collision avoidance manoeuvres occurred }\end{array}$ \\
\hline $\begin{array}{l}\text { Post-mission } \\
\text { disposal }\end{array}$ & $\begin{array}{l}\text { Spacecraft and rocket bodies were moved to orbits that decay within } 25 \text { years (with a } \\
\text { one-year tolerance) or re-orbited above LEO and taken out of the simulation }\end{array}$ \\
\hline
\end{tabular}

\subsection{The DAMAGE Model}

DAMAGE is a three-dimensional computational model capable of predicting the evolution of the full LEO to GEO space debris environment. Recent DAMAGE studies include the effects of thermosphere contractions on the debris population [20] and investigating synergies between ADR and debris mitigation [10].

DAMAGE is supported by a fast, semi-analytical orbital propagator, with a time step of five days, which includes orbital perturbations due to Earth gravity harmonics, $J 2, J 3$, and $J 2,2$, luni-solar gravitational perturbations, solar radiation pressure and atmospheric drag. The drag model assumes a rotating, oblate atmosphere with density and density scale height values taken from the 1972 COSPAR International Reference Atmosphere.

Collisions were predicted using a fast, pair-wise collision prediction algorithm based on the 'Cube' approach adopted in NASA's LEO-to-GEO Environment Debris (LEGEND) model [21], developed in 2003 [22]. The cube size for this collision prediction was $10 \mathrm{~km}$. The NASA standard break-up model, significantly updated in 1998 and published in 2001 [23], was applied when collisions or explosions were detected. Catastrophic collisions occurred when the specific impact energy between the two colliding objects exceeded $40 \mathrm{~J} / \mathrm{g}$ [24]. Whilst the collision algorithm and breakup model are a decade old, they are still in wide use today, such as for the IADC study on the Stability of the Future LEO Environment [11]. 


\subsection{Object Size}

Typically, recent ADR studies have considered objects sized $\geq 10 \mathrm{~cm}[9,10,12]$. This is because these objects are, for the most part, well observed and tracked in LEO and represent approximately $98 \%$ of the total mass in orbit. Additionally, collisions between objects $\geq 10 \mathrm{~cm}$ are more likely to result in catastrophic breakups that lead to the generation of a high number of additional fragments. In contrast, collisions between smaller objects are less likely to generate significant numbers of fragments that globally effect the evolution of the debris environment. Further, limiting the size of objects to $10 \mathrm{~cm}$ restricts the number of propagated objects to the tens of thousands allowing reasonable computational times.

This study however, simulated the $\geq 5 \mathrm{~cm}$ LEO population, allowing the propagation of approximately twice the number of objects compared with the $\geq 10 \mathrm{~cm}$ population. This provided a larger number of collisions to be captured, which may affect ADR activities.

\subsection{Launch Activity}

The number and location of future launches is an essential component of the future collision rate and the evolution of the debris environment [17,19]. In this study, the maximum (129) and minimum (36) yearly launch rates of the last 50 complete years (1962-2012) were used to define the range of future launch rates. A uniformly distributed random number between zero and one, $X_{L} \sim U(0,1)$, was generated for each MC projection. The yearly launch rate, $L$, was

$L=36+93 X_{L}$

where 93 was the range between the maximum and minimum launch rate. The launch rate was rounded to the nearest integer and remained fixed throughout the projection.

For each launched object, six additional numbers were drawn from distributions of the initial orbital elements, along with a further four numbers to determine the object's type (satellite/rocket body), mass, area and size. These values had the same initial orbital elements, mass, area and size distributions of all the objects launched over the last eight complete years (2004-2012). Thus, the properties of future launched objects reflected the current trend of launch activities. This process was then subsequently repeated for each additional projection. Launch statistics and information for this method was acquired from ESA's Database and Information System Characterising Objects in Space [25].

Fig. 1 illustrates the possible launch rates for each projection (shaded region), bounded by the minimum and maximum launch rates (solid black line). The dotted line shows the historical launch rates of the last 50 years. 


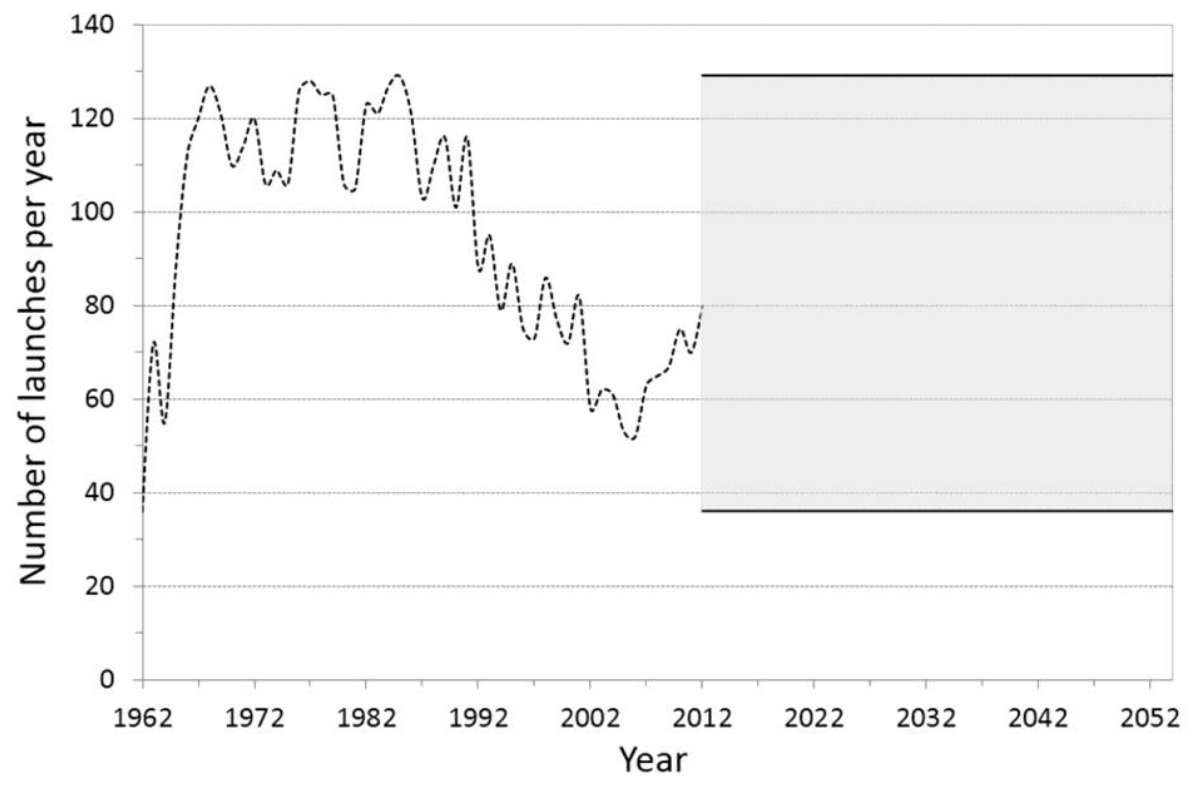

Fig. 1. Historical (dotted line) and possible future launch rates for this study (shaded region).

\subsection{Solar Activity}

Solar activity drives the upper atmospheric density and influences the drag and hence lifetime of debris. Solar irradiance, especially at ultraviolet wavelengths, is the key driver of the mass density change in the thermosphere. The F10.7 cm radio flux values (or F10.7) are often used to link solar activity and upper atmospheric density. Studies, such as $[18,26]$, have shown the important interaction between the long and short-term effects of variations in projected solar activity can have on the LEO debris population.

In this study, projected solar activity was dictated by sine and cosine functions for F10.7 solar cycles. The amplitude of these cycles, $F$, ranged from the largest amplitude $\left(179 \times 10^{-22} \mathrm{~W} \mathrm{~m}^{-2} \mathrm{~Hz}^{-1}\right)$ and smallest amplitude $\left(77 \times 10^{-22} \mathrm{~W} \mathrm{~m}^{-2} \mathrm{~Hz}^{-1}\right)$ solar cycles of the last 50 years. A uniformly distributed random number, $X_{S} \sim U(0,1)$, determined the amplitude of each projection's F10.7 solar cycle

$F=\left(77+112 X_{S}\right) \times 10^{-22}$

The minimum F10.7 value of each solar cycle remained fixed at $71 \times 10^{-22} \mathrm{~W} \mathrm{~m}^{-2} \mathrm{~Hz}^{-1}$, thus only the maximum F10.7 value varied. Throughout each projection, the projected solar cycle amplitude remained fixed. Fig. 2 illustrates this; the dotted line shows the monthly historical F10.7 cm values from 1962-2012. This information was obtained through the National Oceanic and Atmospheric Administration National Geophysical Data Centre [27]. From the beginning of 2013, the shaded region indicates all possible solar cycle amplitude values. Solid lines show the boundaries of these cycles. 


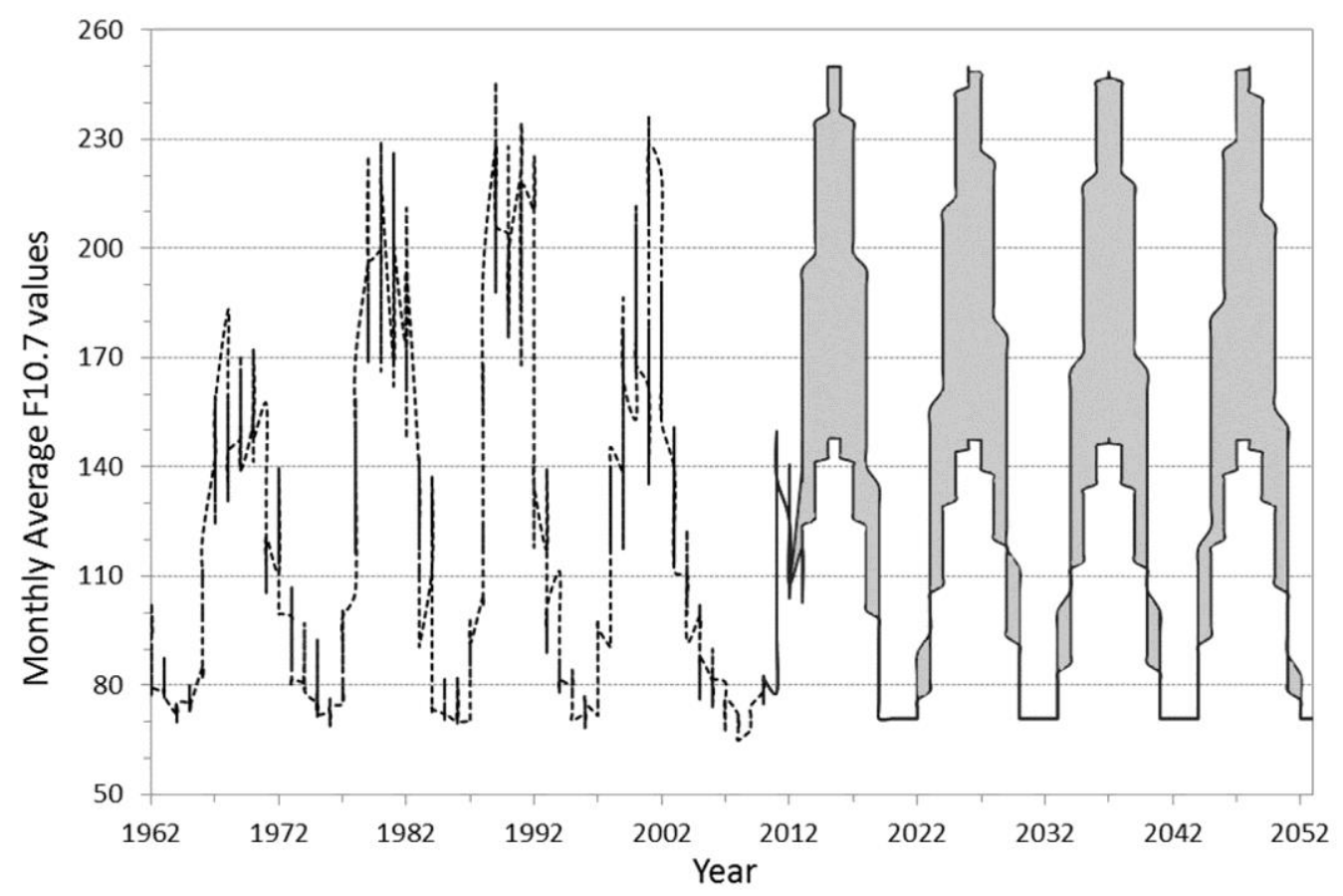

Fig. 2. Historic (dotted line) and possible future monthly F10.7 values for the study (shaded region).

\subsection{Compliance with Post-mission Disposal}

The effectiveness of PMD has been well demonstrated $[6,7,10,16]$. Some recent ADR studies have used future PMD compliances levels of $90 \%$ with a 25 -year de-orbit time $[9,10,12]$. However, in 2010, based on early data, it was estimated that less than $14 \%$ of spacecraft and rocket bodies reaching their end-of-life in the critical altitudes of 600-1,400 km reduced their altitude to comply with this. Since the completion of this work this value of $14 \%$ has been re-evaluated to $8 \%$ [28].

Compliance with PMD in this study ranged between $14-90 \%$ for objects launched after 2009. This reflected the estimated compliance in 2010 and an optimistic future case. The fraction of objects complying with PMD, $P$, for each projection was calculated using a uniformly distributed random number, $X_{p} \sim U(0,1)$ such that

$P=0.14+0.76 X_{P}$

this value remained fixed for the duration of the projection, until the process was repeated. Objects complying with PMD were manoeuvred to orbits that decayed within 25 years (with a one-year tolerance) or re-orbited above LEO and taken out of the projection.

\subsection{Explosion Activity}


To model the variation in explosion activity, the highest (5 explosions) and the lowest (no explosions) yearly observed explosion rate were taken from the past 50 years. A uniformly distributed random number, $X_{E} \sim U(0,1)$, determined the yearly explosion rate, $E$, between these two values

$E=5 X_{E}$,

the explosion rate was rounded to the nearest integer and remained fixed for the projection. The orbital elements, object type and mass were all calculated with a further eight numbers that had the same distributions of the orbital elements, object type and mass, of explosions that occurred over the last eight complete years (2004-2012).

\subsection{Active Debris Removal}

The ADR target selection criterion used by DAMAGE, $R_{i}(t)$, based on minimising the risks of a collision [28], was

$R_{i}(t)=P_{i}(t) \times m_{i}$

where $m_{i}$ is the mass of object $i$ and $P_{i}(t)$ is the total collision probability of object $i$ at a specific time $t$. Objects with the highest mass-collision probability were removed at the beginning of each year after 2020 . As well as this criterion, the objects removed were intact, had an orbital eccentricity $<0.5$, perigee altitude $<$ $1,400 \mathrm{~km}$ and were removed immediately from the projection.

\subsection{Performance Metrics}

An Effective Reduction Factor (ERF) [29] was calculated to quantify the effect of ADR. The ERF is defined as the number of debris objects reduced in the environment over a period $t$ using ADR, divided by the total number of removed objects

$E R F(t)=\frac{N(t)-N_{S}(t)}{C N_{R}(t)}$

Where $N(t)$ is the effective number of objects $\geq 10 \mathrm{~cm}$ or $\geq 5 \mathrm{~cm}$ in a scenario with no ADR (ADR0), $N_{s}(t)$ is the effective number of objects $\geq 10 \mathrm{~cm}$ or $\geq 5 \mathrm{~cm}$ for an ADR scenario, and $C N_{R}(t)$ is the cumulative number of objects removed for the ADR scenario by time $t$. The ERF was measured as the end of the projection in 2209. 
The size of the $\geq 5 \mathrm{~cm}$ and $\geq 10 \mathrm{~cm}$ LEO populations, as well as the cumulative number of collisions (catastrophic and non-catastrophic) were recorded and analysed for each projection.

\section{Results and Discussion}

Graphically, the arithmetic average of each scenario's debris populations provides a straightforward way to interpret key future trends. The $200 \mathrm{MC}$ arithmetic average of the LEO debris population for each scenario is presented in Fig. 3 for the $\geq 5 \mathrm{~cm}$ population and Fig. 4 for the $\geq 10 \mathrm{~cm}$ population. Further statistical information including the standard deviations of these populations is shown in Table 2.

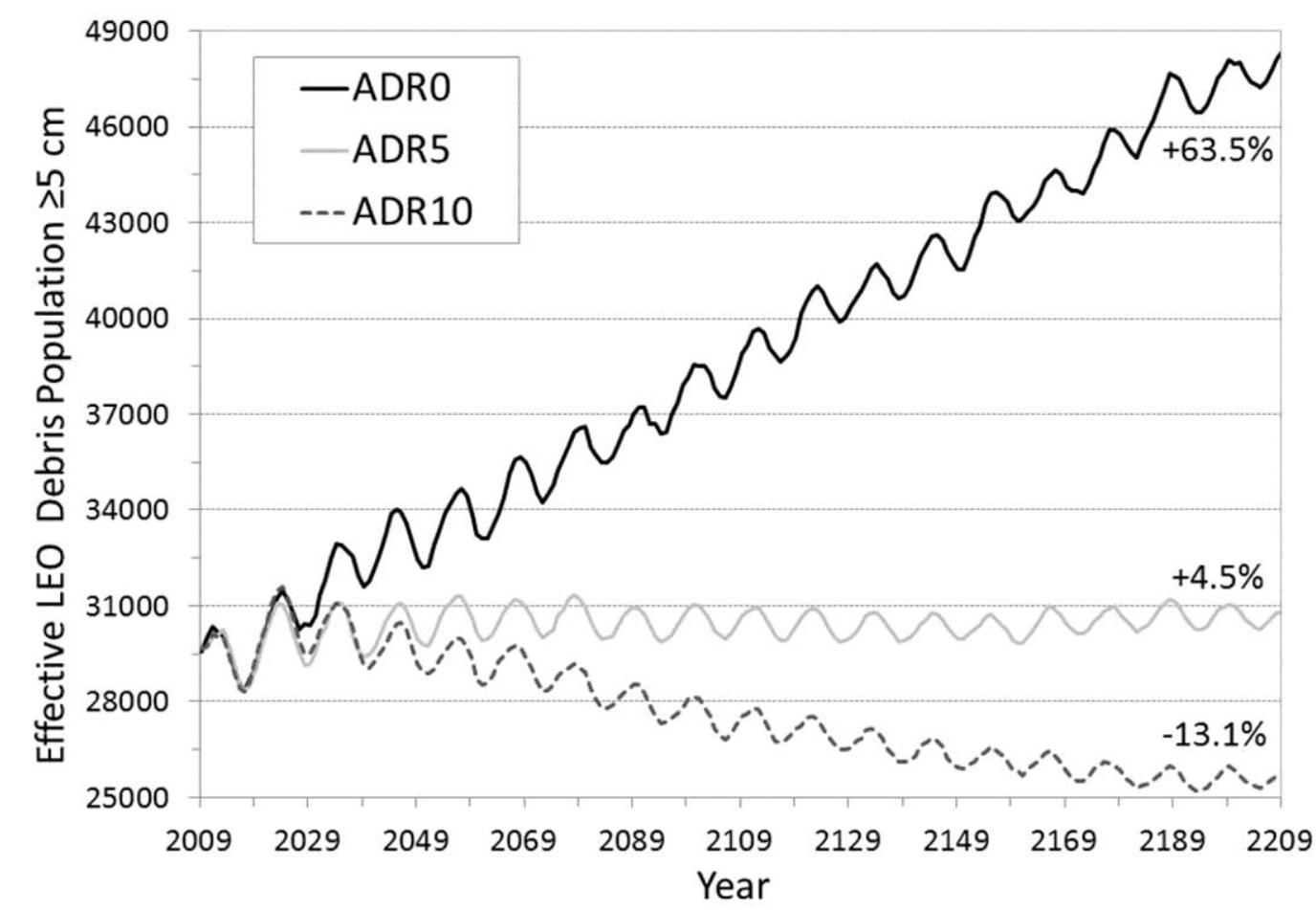

Fig. 3. 200 MC arithmetic average of objects $\geq 5 \mathrm{~cm}$ in LEO for each scenario. Percentage values indicate the percentage change in the 2209 population compared with the initial population in 2009. 


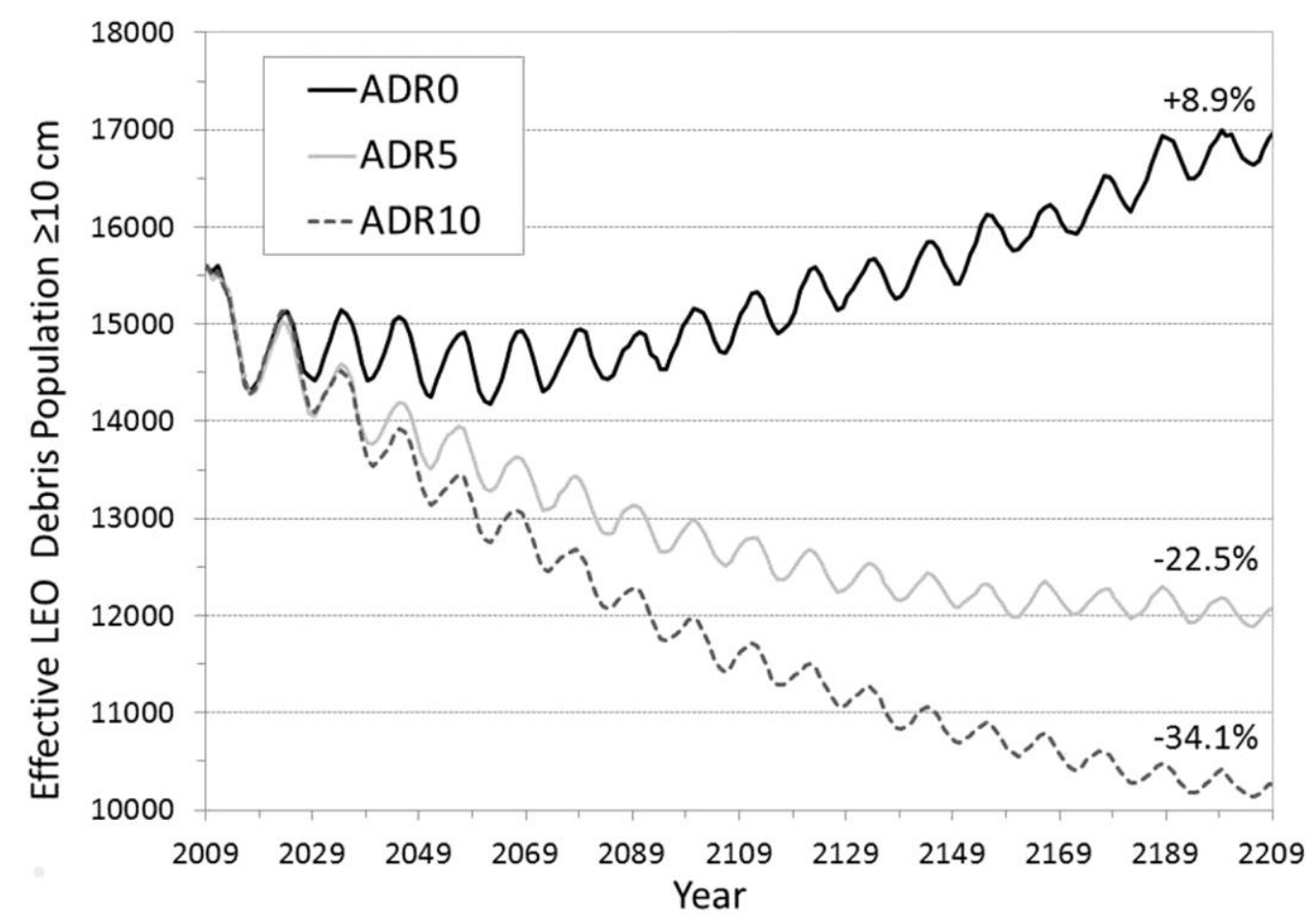

Fig. 4. 200 MC arithmetic average of objects $\geq 10 \mathrm{~cm}$ in LEO for each scenario. Percentage values indicate the percentage change in the 2209 population compared with the initial population in 2009.

In the ADR0 scenario, the average population increased, though, the $\geq 5 \mathrm{~cm}$ percentage population increase was over seven times larger than that of the $\geq 10 \mathrm{~cm}$ population. This greater increase was driven by a tripling of objects sized 5-10 cm, which represent the generation of thousands of new fragments from each catastrophic collision or explosion. This presents a concern to satellite operators: whilst a $9 \%$ growth in $\geq 10$ $\mathrm{cm}$ population may be manageable, a $224 \%$ growth in $5-10 \mathrm{~cm}$ objects may pose a major collision risk, particularly as these sized objects are currently difficult to observe and track.

Removing debris by ADR reduced the average LEO population; this conforms to previous ADR studies. Active debris removal had a larger effect on reducing the $\geq 5 \mathrm{~cm}$ population compared with $\geq 10 \mathrm{~cm}$ population (see ERF values in Table 2). However, removing five objects per year stopped the growth of $\geq 10$ $\mathrm{cm}$ objects in $83 \%$ of projections, but only $49 \%$ of projections for populations of objects sized $\geq 5 \mathrm{~cm}$. This suggests more removals will be required to stop the $5-10 \mathrm{~cm}$ population from increasing than the $\geq 10 \mathrm{~cm}$ population. Even in the case of 10 removals, in this study, there remained a $32 \%$ chance that the $\geq 5 \mathrm{~cm}$ population would continue to grow. 
Table 2. Summary of the 200 MC results for each scenario at the 2209 epoch.

\begin{tabular}{|c|c|c|c|c|c|c|}
\hline \multirow[t]{2}{*}{ Scenario } & \multicolumn{2}{|c|}{$\begin{array}{c}\text { Standard deviation of the } \\
\text { population at the } 2209 \text { epoch }\end{array}$} & \multicolumn{2}{|c|}{$\begin{array}{l}\text { Percentage of MC's end population } \\
\text { greater than initial population (\%) }\end{array}$} & \multicolumn{2}{|c|}{ Average ERF } \\
\hline & $\geq 5 \mathrm{~cm}$ & $\geq 10 \mathrm{~cm}$ & $\geq 5 \mathrm{~cm}$ & $\geq 10 \mathrm{~cm}$ & $\geq 5 \mathrm{~cm}$ & $\geq 10 \mathrm{~cm}$ \\
\hline ADR0 & 16,70 & 5030 & 70 & 54 & - & \\
\hline ADR5 & 11,367 & 3662 & 51 & 17 & 18.3 & 5.2 \\
\hline ADR10 & 10,817 & 3626 & 32 & 6 & 11.9 & 3.6 \\
\hline
\end{tabular}

Increasing the removal rate, from five to 10 removals per year, reduced the overall effectiveness of ADR; as demonstrated by the ERF values in Table 2. Doubling the removal rate reduced the ERF values by $30-40 \%$. This implies that the more removals that are made the less efficient ADR becomes at reducing the total debris population; this is summarised in Fig 5. This figure shows the average values of the top 10 ranked object's (x-axis) target selection criterion, $R_{i}(t)$, over the projection period. The more objects that were removed the less effect each individual object had on reducing the debris population.

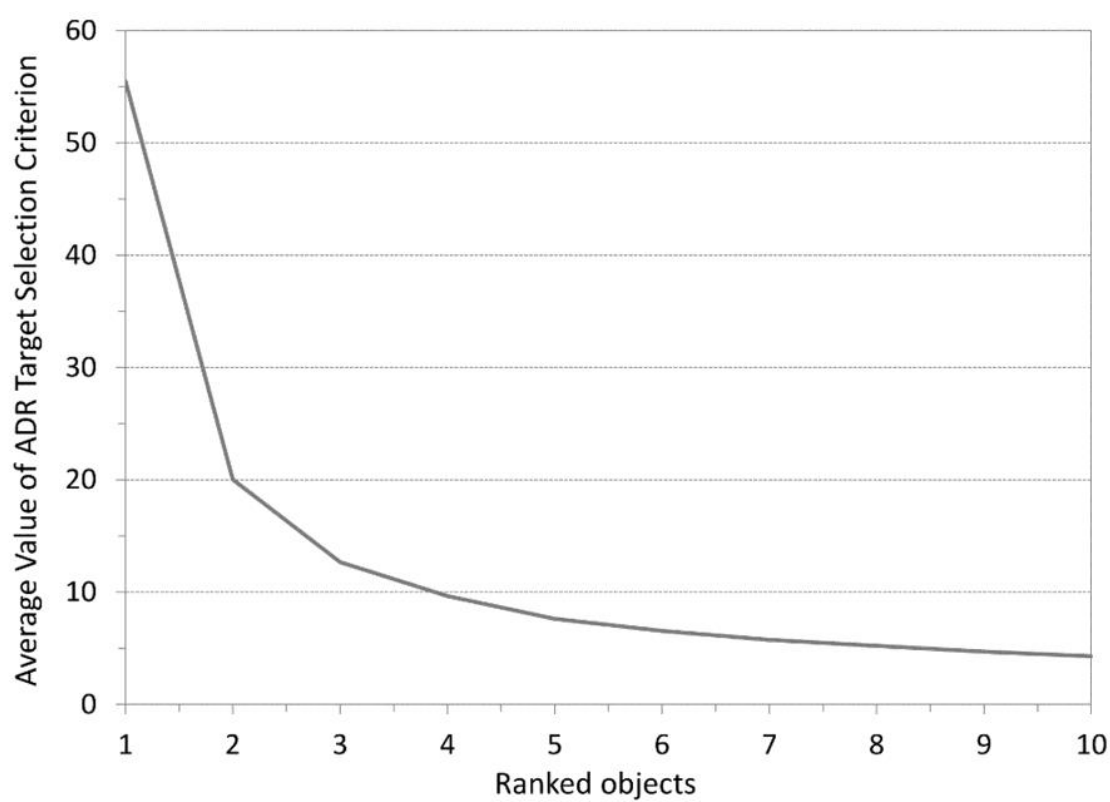

Fig. 5. The average ADR target selection values for the top ten objects removed each year, for one MC simulation in the ADR10 scenario.

The average number of collisions for each scenario (Table 3) follows a similar trend to the total number of objects. Approximately $45 \%$ of collisions involved at least one object $<10 \mathrm{~cm}$ in size; simulating the $5-10 \mathrm{~cm}$ population increases the total number of collisions by almost a factor of two compared with just modelling the $\geq 10 \mathrm{~cm}$ population. Typically these collisions would involve an $<10 \mathrm{~cm}$ impactor (the less massive of the two colliding objects) and $\mathrm{a} \geq 10 \mathrm{~cm}$ target object. Whilst this is a major proportion of the total number of collisions, only 1.8-3.5 collisions (depending upon the scenario) of these were catastrophic. This is because 
the smaller sized impactors, usually do not carry a high enough mass to exceed a specific impact energy of $40 \mathrm{~J} / \mathrm{g}$. Despite this, collisions caused by $5-10 \mathrm{~cm}$ objects had an impact on the total number of objects $\geq 10$ $\mathrm{cm}$.

Table 3. The average number and proportions of collisions, separated by type and impactor size, for each scenario

\begin{tabular}{|c|c|c|c|c|c|}
\hline \multirow{3}{*}{ Scenario } & \multirow{3}{*}{$\begin{array}{l}\text { Average cumulative } \\
\text { number of collisions }\end{array}$} & \multicolumn{4}{|c|}{ Percentage of collisions $(\%)$ that were: } \\
\hline & & \multicolumn{2}{|c|}{ Non-catastrophic } & \multicolumn{2}{|c|}{ Catastrophic } \\
\hline & & $\begin{array}{l}<10 \mathrm{~cm} \\
\text { impactor }\end{array}$ & $\begin{array}{l}\geq 10 \mathrm{~cm} \\
\text { impactor }\end{array}$ & $\begin{array}{l}<10 \mathrm{~cm} \\
\text { impactor }\end{array}$ & $\begin{array}{l}\geq 10 \mathrm{~cm} \\
\text { impactor }\end{array}$ \\
\hline ADR0 & 77.6 & 40.7 & 24.9 & 4.5 & 29.9 \\
\hline ADR5 & 40.3 & 38.9 & 18.9 & 5.5 & 36.5 \\
\hline ADR10 & 31.3 & 38.4 & 19.3 & 5.7 & 36.5 \\
\hline
\end{tabular}

Only showing results of the average debris populations, such as in Fig. 3-4, loses information on individual projections. In this study, all projections were unique in there four key varied parameters, and therefore it is vital to present their results accordingly. Fig. 6 displays a histogram of all the individual MC projections LEO debris $\geq 5 \mathrm{~cm}$ populations after 200 years in bins, of size 5,000 objects. Each bin represents the proportion of MC projections that had a particular debris population at 2209 epoch. This histogram therefore illustrates the distribution of debris populations for each scenario. For each scenario, the size of the population range was over a factor of 10. The shaded portion of Fig.6 indicates the region where the population had grown in comparison to the initial population (expressed as percentage values in Table 2). 


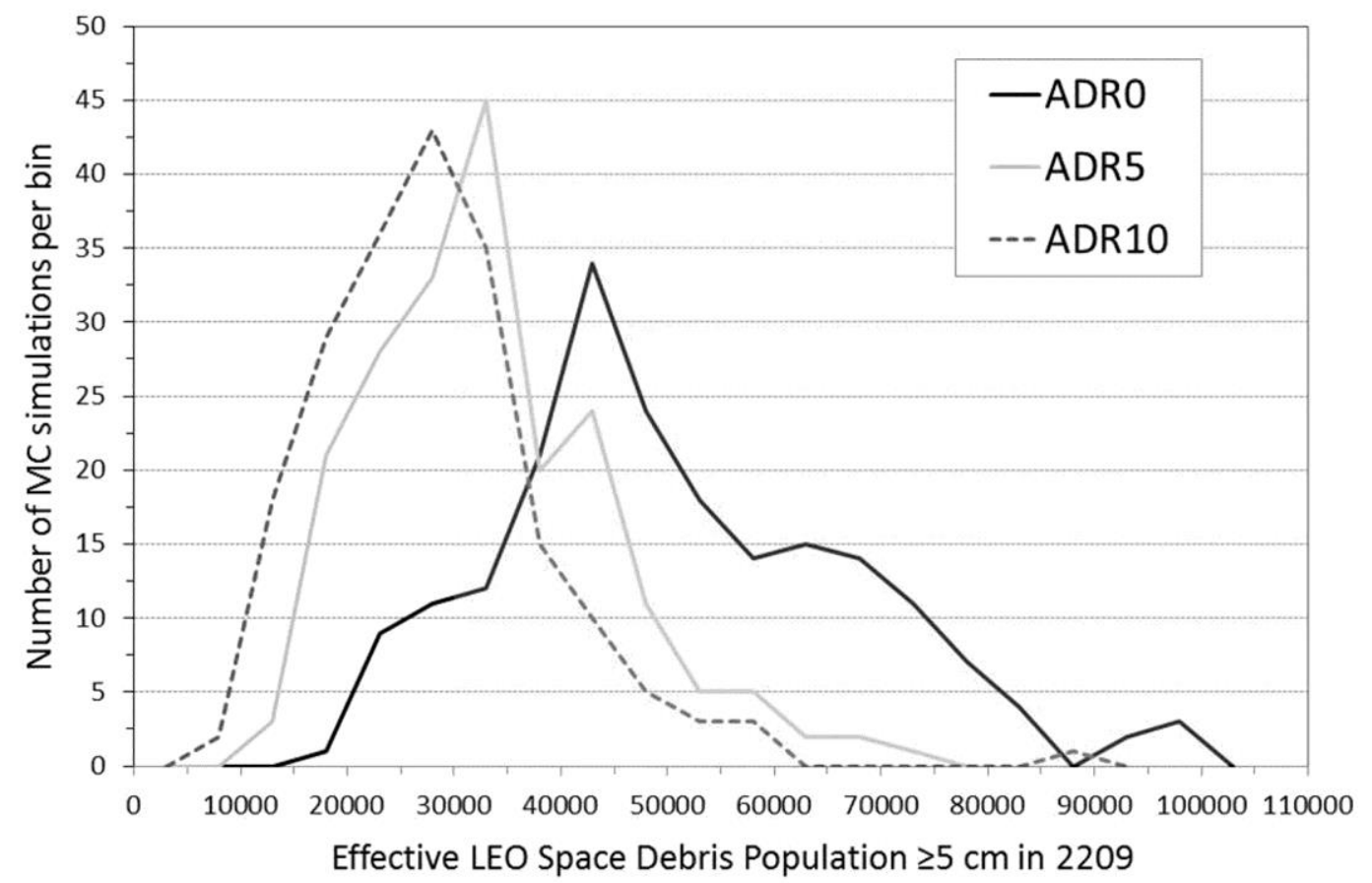

Fig. 6. Histogram of the effective LEO debris population $\geq 5 \mathrm{~cm}$ of each simulation. Bin size equal to 5,000 objects.

In all the scenarios, there is a considerable region where two or more scenarios population distributions intersect and the area under each distribution overlaps with the area of a different scenario. This overlap shows the region where two or more scenarios had the same final population. In all three scenarios, there is overlap, expressed as a percentage of the total area of distribution; this was 52\% between ADR5 and ADR0, $36 \%$ between ADR10 and ADR0 and 58\% between ADR5 and ADR10. The region of overlap shows where performing ADR or increasing the removal rate has not had any additional benefit, because the value of the modelled parameters, representing launch, explosion, solar and mitigation activity, had a larger influence on the evolution of the debris environment. This therefore shows that the values of parameters can offset the positive effect of ADR. Conversely, depending upon the values of these parameters, ADR is not always required in order to prevent the debris population. This is shown by the fact that the final population in $30 \%$ of ADR0 projections had reduced.

The distribution of populations in each scenario in Fig. 6 does not fit a Gaussian distribution. The coefficient of determination $\left(R^{2}\right)$, ranged between 0.63-0.68 for a fitted Gaussian. Instead, a better distribution was found to be log-normal, with higher values of coefficients of determination between 0.890.91. Using the data in Fig. 6, a log-normal probability density function as a function of the final number of objects, $f(N)$, can be calculated as 
$f(N)=a \times e^{-\frac{\left(\frac{\ln (N)-b}{c}\right)^{2}}{2}}$

where $a, b$ and $c$ are coefficients of the log-normal distribution for a bin size of 5,000 objects and $N$ is the total number of objects at the end of the 200 -year projection. The coefficients and statistics for each distribution are given in Table 4.

Table 4. Coefficients of log-normal distributions for objects $\geq 5 \mathrm{~cm}$, their correlation coefficients and statistics.

\begin{tabular}{lllllllll}
\hline Scenario & a & b & c & $\boldsymbol{R}^{\mathbf{2}}$ & Mean & Mode & Median & $\begin{array}{l}\text { Standard } \\
\text { deviation }\end{array}$ \\
\hline ADR0 & 0.13 & 10.69 & -0.34 & 0.88 & 46,528 & 39,120 & 43,915 & 16,288 \\
ADR5 & 0.19 & 10.26 & -0.35 & 0.93 & 30,371 & 25,273 & 28,567 & 10,964 \\
ADR10 & 0.20 & 10.10 & 0.39 & 0.91 & 26,226 & 20,973 & 24,343 & 10,512 \\
\hline
\end{tabular}

As the results are not a Gaussian distribution, the arithmetic mean populations shown in Fig. 3 and Fig. 4 do not represent the most likely (the mode) future population. In the case of the log-normal distribution, the mode population is $15-25 \%$ lower than the arithmetic mean population. This suggests, in this case, designing ADR requirements based on the arithmetic mean of modelling results (like Fig. 3 and Fig. 4 ) is a more conservative approach than designing for the mode.

To give an indication of each parameter's effect on the total number of debris objects, multiple linear regression was used. The total number of objects $\geq 5 \mathrm{~cm}$ after a 200 year projection, $N$, can be approximated as

$N=k_{1}+k_{2} L+k_{3} F+k_{4} P+k_{5} E+k_{6} N_{R}$

$N_{R}$ is equal to the number of objects removed by ADR each year. Each parameter (launch traffic, solar activity etc.) has been normalised to enable the coefficients of multiple linear regression, $k_{1}$ through $k_{6}$, to show the population difference between the worst and best-case (Table 5).

Table 5. Coefficients of multiple linear regression for objects $\geq 5 \mathrm{~cm}$ and their coefficients of determination.

\begin{tabular}{lllllllll}
\hline & & Launch & Solar & \multicolumn{2}{l}{ Compliance } & Explosion & \multirow{2}{*}{ ADR } & $\begin{array}{l}\text { Coefficient of } \\
\text { Scenario }\end{array}$ \\
& $\boldsymbol{k}_{\mathbf{1}}$ & activity & activity & with & PMD & activity & $\begin{array}{l}\left(\boldsymbol{k}_{\mathbf{6}}\right) \\
\text { determination }\end{array}$ \\
& & $\left(\boldsymbol{k}_{\mathbf{2}}\right)$ & $\left(\boldsymbol{k}_{\mathbf{3}}\right)$ & $\left(\boldsymbol{k}_{\mathbf{4}}\right)$ & $\left(\boldsymbol{k}_{\mathbf{5}}\right)$ & & $\left(\boldsymbol{R}^{\mathbf{2}}\right)$ \\
\hline ADR0 & 60,563 & 4,767 & $-39,820$ & $-2,241$ & 16,204 & - & 0.57 \\
ADR5 & 33,743 & 6,047 & $-23,555$ & $-3,987$ & 18,189 & - & 0.71
\end{tabular}




$\begin{array}{llllllll}\text { ADR10 } & 29,089 & 6,066 & -23,846 & -3,090 & 18,172 & - & 0.74 \\ \text { All scenarios } & 52,498 & 5,401 & -28,727 & -3,664 & 17,093 & 21,839 & 0.79 \\ \text { combined } & & & & & & & \end{array}$

This type of analysis is a coarse estimation of the total number of objects, as it assumes each variable is independent and linear and that no other factors contribute to the population. The effectiveness of PMD compliance however, will be dependent upon the number of objects launched. Yet, $R^{2}$ values associated with these regressions (Table 5) show enough of a fit to approximate which parameters have the greatest effect on the size of the population. In order of biggest to smallest effect these were, solar activity, ADR, explosion activity, launch activity and finally PMD compliance. This appears to be a direct contradiction of results in [30], which has shown that PMD compliance has a greater effect on the LEO debris population than passivation (explosion activity). The probable reasons for this is that [30] was run with a population size $\geq 10$ $\mathrm{cm}$ and an eight-year repeatable explosion cycle, compared with this study's $\geq 5 \mathrm{~cm}$ population and variable explosion activity. Furthermore, according to this study the overall effectiveness of PMD compliance will vary dependently on the launch activity in a given projection.

\subsection{Validating the Number of Monte Carlo Projections}

To determine if 200 projections were reliable enough to establish the trends and distributions of the results, a sub-sampling technique was applied, introduced by [31]. This technique was modified to allow the standard deviation to be analysed instead of the arithmetic mean. In doing so, this allows the statistical dispersion between projections to be analysed. The procedure for this analysis was

- Starting with $Z=1$, randomly select $Z$ number of MC projections (out of $200 \mathrm{MCs}$ )

- Calculate the standard deviation of the debris population at the end of the projection for the selected MCs

- Repeat the previous two steps with the same value of $Z, 200$ times

- Repeat the previous three steps incrementing $Z$ by one until $Z=100$

Fig. 7 illustrates this process for the ADR0 scenario. The $\mathrm{x}$-axis represents the values of $Z$ between 0 100. At each $Z$ value, 200 individual values are plotted. Each point represents the standard deviation from a random selection of $Z$ samples. The $200 \mathrm{MC}$ standard deviation and $20 \%$ from this deviation (dark solid lines) are shown. 


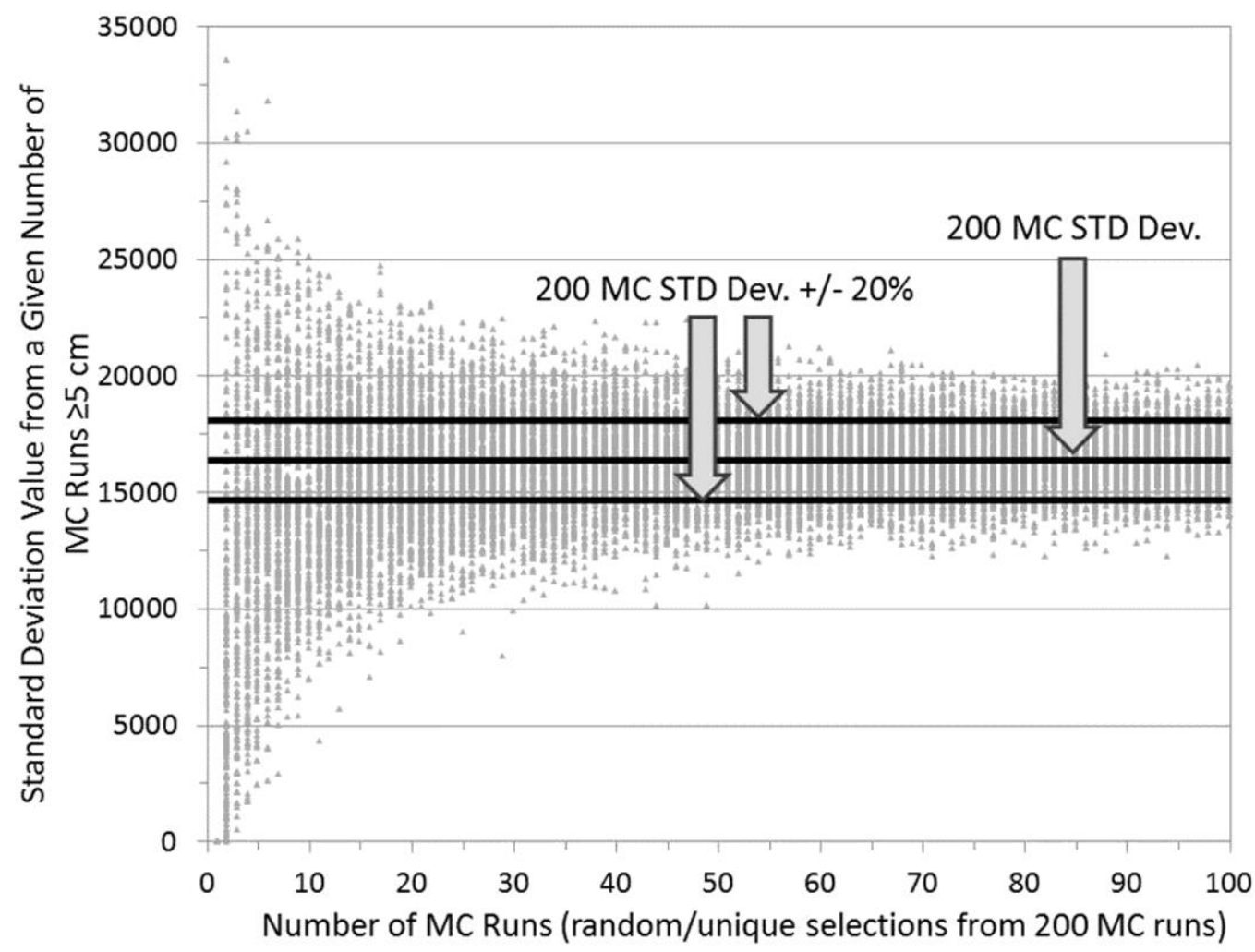

Fig. 7. Sub-sampled standard deviations from 200 MC projections of the $\geq 5 \mathrm{~cm}$ LEO debris populations in 2209.

Both ADR5 and ADR10 scenarios show a very similar trend to ADR0 in Fig. 7. To achieve a standard deviation within $20 \%$ of the $200 \mathrm{MC}$ "true" standard deviation nine out of 10 times, 71 projections must be run for ADR0, 69 for ADR5 and $66 \mathrm{MCs}$ for ADR10. Beyond 80 projections, the improvements start to decline. Thus $200 \mathrm{MCs}$ for each scenario, in this case gave an acceptable result to draw conclusions about the study.

\section{General Discussion}

Active debris removal is an effective measure to help control the LEO population. Results show, however, that it is not possible to rely alone, on removing 10 objects per year to prevent the growth of the $\geq 5$ or $\geq 10$ $\mathrm{cm}$ population. Launch, solar, mitigation and explosion activity can offset or have a larger influence on how the population evolves compared with performing ADR. Particularly, as the efficiency of ADR reduces with increasing removal rate. This can be seen with the $36-58 \%$ population overlap between scenarios in Fig. 6 , and the range of debris populations in each scenario differing by a factor of 10 .

Nevertheless, launch activity, compliance with PMD and explosion activity are all parameters we can, in theory, control. It can be argued that, with the growing momentum and further implementation of guidelines, standards and legal practices, the expected number of objects that will comply with PMD guidelines will 
increase, and the number of orbital explosions will decrease; although, this cannot be guaranteed. For this study, a uniform distribution of values for each parameter was selected in order to investigate and analysis as wide a range of potential future cases as possible. Despite using this uniform distribution, the final populations of the projections were found to better fit a log-normal distribution than a Gaussian. This shifts the mode debris population lower than the arithmetic mean population.

Debris sized between five and $10 \mathrm{~cm}$ is likely to grow far more rapidly than objects $\geq 10 \mathrm{~cm}$ in size. As a result, it is probable a higher number of removals will be required to reduce the growth of this population size compared with the $\geq 10 \mathrm{~cm}$ population. This smaller sized debris poses a greater short-term risk to satellite operators, as impacts of this size can lead to the partial or complete loss of a satellite. The number of removals and high-level objectives of ADR, therefore, will depend upon the importance of this short-term risk.

In order to meet specific objectives concerning the future debris environment (such as limited or zero growth in the LEO population), the required number of removals performed by ADR will be dependent, in part, upon the future values of these parameters. The exact values of these parameters will always remain uncertain. Other future uncertainties, such as advances in technology or differing socio-economic drivers, may also change requirements for ADR activities.

Further, the methods of debris models and the way they are developed and implemented affect the results of long-term predictions. For example, different collision detection algorithms and breakup models, such as those in [32], may give different prediction results; even if other parameters remain the same.

Recent results by [33], comparing population predictions of objects greater than one $\mathrm{cm}$, have shown similar trends, although absolute numbers of objects differ between the two predictions. This is likely to be because of the differing collision detection algorithms and breakup models implemented within the two models. It is important that different implementations of debris models form part of the investigation of the future debris environment, so that that consensus may be reached on appropriate mitigation and remediation measures.

Given all this, one method to determine removal rates for ADR, implemented by [34], is to react and adapt to the evolution of space debris. This study ([34]) has shown that, by monitoring the behaviour of each MC projection and adjusting, at regular intervals, the ADR rate within the projection, it is possible to increase drastically the probability of achieving a desired LEO population. This approach has made ADR more efficient (increasing the ERF), and required fewer removals per year than a fixed removal rate.

\section{Conclusion}

The purpose of this paper was to highlight some variability in parameters used to model the long-term evolution of the LEO debris environment, and to show their effect on ADR activities. Active debris removal is a positive proactive measure for reducing the long-term LEO population. However, the results have shown 
that depending on the values of these parameters a population difference of over a factor of 10 between projections can be observed. It is recommended that continued studies by nations, committees and others in order to determine the necessity and full requirements for ADR.

\section{Acknowledgements}

Financial support for this work was provided by the University of Southampton's Faculty of Engineering and the Environment. The authors would like to thank Holger Krag (ESA, Space Debris Office) for permission to use the MASTER population data.

\section{References}

[1] Inter-Agency Space Debris Co-ordination Committee, IADC Space Debris Mitigation Guidelines, IADC-02-01, 2007, available at http://www.iadc-online.org.

[2] Scientific and Technical Subcommittee of the Committee on the Peaceful Uses of Outer Space, Space Debris Mitigation Guidelines of the Scientific and Technical Subcommittee of the Committee on the Peaceful Uses of Outer Space, A/AC.105/890, 2008, Annex IV (endorsed under UN Resolution $\mathrm{A} / \mathrm{RES} / 62 / 217)$.

[3] Kato, A. Lazare, B. Oltrogge, D. Stokes, P.H. Standardization By ISO to Ensure the Sustainability of Space Activities, Proceedings of the Sixth European Conference on Space Debris, ESOC, Darmstadt, Germany, 22-25 April 2013, European Space Agency Publication SP-723.

[4] H. Klinkrad, P. Beltrami, S. Hauptmann, C. Martin, H. Sdunnus, H. Stokes, R. Walker, J. Wilkinson, The ESA Space Debris Mitigation Handbook 2002, Adv. Space Res., 34 (5) (2004), pp. 1251-1259.

[5] L. Anselmo, A. Rossi, C. Pardini, A. Cordelli, R. Jehn, 2001, Effect of Mitigation Measures on the Long-term Evolution of the Debris Population, Adv. Space Res., 28 (9) (2001), pp. 1427-1436.

[6] R. Walker, C.E. Martin, P.H. Stokes, J.E. Wilkinson, H. Klinkrad, Analysis of the Effectiveness of Space Debris Mitigation Measures using the DELTA Model, Adv. Space Res., 28 (9) (2001), pp. 14371445 .

[7] P.H. Krisko, N.L. Johnson, J.N. Opiela, EVOLVE 4.0 Orbital Debris Mitigation Studies, Adv. Space Res., 28 (9) (2001), pp.1385-1390. 
[8] J.-C. Liou, N.L. Johnson, Risks in Space from Orbiting Bebris, Science, 311 (2006), pp. 340-341.

[9] J.-C. Liou, N.L. Johnson, Instability of the Present LEO Satellite Populations, Adv. Space Res., 41 (7) (2008), pp. 1046-1053.

[10] H.G. Lewis, A.E. White, R. Crowther, P.H. Stokes, Synergy of Debris Mitigation and Removal, Acta Astronautica, 81 (1) (2012), pp. 62-68.

[11] Inter-Agency Space Debris Co-ordination Committee, Stability of the Future LEO Environment, IADC-12-08, 2013, available at http://www.iadc-online.org.

[12] J.-C. Liou, N.L. Johnson, N.M. Hill, Controlling the Growth of Future LEO Debris Populations with Active Debris Removal, Acta Astronautica, 66 (5-6) (2010), pp. 648-653.

[13] H. Klinkrad, N.L. Johnson, Space Debris Environment Remediation, IAA Cosmic Study, International Academy of Astronautics, Paris, France, 2010.

[14] Lewis, H.G. Swinerd, G.G. Newland, R.J. Saunders, A. Active Removal Study for On-orbit Debris using DAMAGE, Proceedings of the Fifth European Conference on Space Debris, ESOC, Darmstadt, Germany, 30 March-2 April 2009, European Space Agency Publication SP-672.

[15] Liou, J.-C. Challenges and Opportunities for Orbital Debris Environment Remediation, Second European Workshop on Active Debris Removal, CNES HQ, Paris, France, 18-19 July 2012.

[16] J.-C. Liou, N.L. Johnson, A LEO Satellite Postmission Disposal Study using LEGEND, Acta Astronautica, 57 (2-8) (2005), pp. 324-329.

[17] C. Martin, R. Walker, H. Klinkrad, The Sensitivity of the ESA DELTA Model, Adv. Space Res. 34 (5) (2004), pp. 969-974.

[18] M. Horstman, Varying Solar Flux Models and their Effect on the Future Debris Environment Projection, Orbital Debris Quarterly News, Vol. 9, No. 1 NASA Johnson Space Center, Houston, TX, 2005, pp.4. 
[19] P. Eichler, H. Sdunnus, J. Zhang, Reliability of Space Debris Modelling and the Impact of Current and Future Space Flight Activities, Adv. Space Res, 13, (8) (1993), pp. 225-228.

[20] H.G. Lewis, A. Saunders, G. Swinerd, R.J. Newland, Effect of Thermospheric Contraction on Remediation of the Near-Earth Space Debris Environment, J. Geophys. Res., 116 (2011), A00H08, doi:10.1029/2011JA016482.

[21] J.-C. Liou, D.T. Hall, P.H. Krisko, J.N. Opiela, LEGEND — a three-dimensional LEO-to-GEO Debris Evolutionary Model, Adv. Space Res., 34 (5) (2004), pp. 981-986.

[22] Liou, J.-C. Kessler, D. J. Matney, M. Stansbery, G. A New Approach to Evaluate Collision Probabilities Among Asteroids, Comets, and Kuiper Belt Objects, 34th Annual Lunar and Planetary Science Conference, 17-21 March 2003, League City, Texas, abstract no.1828.

[23] N.L. Johnson, P.H. Krisko, J.-C. Liou, P.D. Anz-Meador, NASA's new Breakup Model of EVOLVE 4.0, Adv. Space Res., 28 (9) (2001), pp. 1377-1384.

[24] McKnight, D.S. Collision and Breakup Models: Pedigree, Regimes and Validation/Verification, Briefing presented to the National Research Council Committee on Space Debris Workshop, Irvine, California, November 1993.

[25] Flohrer, T. Lemmens, S. Bastida Virgili, B. Krag, H. Klinkrad, H. Parrilla, E. Sanchez, N. Oliveira, J. Pina, F. DISCOS- Current Status and Future Developments, Proceedings of the Sixth European Conference on Space Debris, ESOC, Darmstadt, Germany, 22-25 April 2013, European Space Agency Publication SP723.

[26] D. Whitlock, Modelling the Effect of High Solar Activity on the Orbital Debris Environment, in: Orbital Debris Quarterly News, vol. 10, No. 2, NASA Johnson Space Centre, Houston, TX, 2006, pp. 4.

[27] National Oceanic and Atmospheric Administration National Geophysical Data Centre, 2013, available at http://www.swpc.noaa.gov/ (last accessed: 12/09/13).

[28] Krag, H. Flohrer, T. Stijn, S. Consideration of Space Debris Mitigation Requirements in the Operation of LEO Missions. 2012 SpaceOps Conference, 11-15 June 2012, Stockholm, Sweden, Paper number: 1257086. 
[29] J.-C. Liou, N.L. Johnson, A Sensitivity Study of the Effectiveness of Active Debris Removal in LEO, Acta Astronauta, 64 (2-3) (2009), pp. 236-243.

[30] White, A.E. Lewis, H.G. Stokes, P.H. The Effectiveness of Space Debris Mitigation Measures, International Space University 16th Annual Symposium, International Space University, Strasbourg, France, 21-23 Feb 2012.

[31] J.-C. Liou, A Statistical Analysis of the Future Debris Environment, Acta Astronautica, 62 (2) (2008), pp. 264-271.

[32] N.N. Smirnov (Ed.), Space Debris Hazard Evaluation and Mitigation, Taylor and Francis, London, New York (2002).

[33] Nazarenko, A.I. Usovik, I.V. Space Debris Environment Modeling with Allowance of Mutual Collisions of Objects Larger than $1 \mathrm{~cm}$ in Size, Proceedings of the Sixth European Conference on Space Debris, ESOC, Darmstadt, Germany, 22-25 April 2013, European Space Agency Publication SP723.

[34] White, A.E. Lewis, H.G. Adaptive Strategies for Space Debris Mitigation and Remediation, 63rd International Astronautical Congress, Naples, Italy, 1-5 Oct 2012, Article: IAC-12-A6.2.20. 